Results There were 3 males and two females. Prenatal diagnosis was made in 4 cases. There were 4 full's term newborns and one near term of 36 weeks. Pleural effusion was on the right side in three cases, on the left side in one case and bilateral in one case. Four cases required mechanical ventilation. Somatostatin was indicated in one case. The treatement was successful in four cases. One case presented a dysmorphic syndrome was died by pneumothoracis.

Conclusions The treatment of congenital chylothorax is based on conservative management. Somatostatin or its analog octreotide are considered as an adjunctive treatement of congenital chylothorax. However, the refractory cases are treated with chemical pleurodenis or surgical treatment. We propose an algorithm of the treatment of congenital chylothorax after review of the literature.

\section{P640 NEONATAL ARRHYTHMIAS: A 20 CASES STUDY}

${ }^{1}$ Manel Charfi', 'Amel Ben Hamad, ${ }^{1}$ Mouna Loukil, ${ }^{1}$ Chiraz Regaieg, 'Amira Bouraoui, ${ }^{1}$ Ridha Regaieg, ${ }^{1}$ Nedia Hmida, ${ }^{2}$ Dorra Abid, ${ }^{2}$ Samir Kammoun, ${ }^{1}$ Afef Ben Thabet, ${ }^{1}$ Abdellatif Gargouri. 'Department of Neonatology, Hedi Chaker University Hospital, Sfax, Tunisia; ${ }^{2}$ Department of Cardiology Hedi Chaker University Hospital, Sfax, Tunisia

\subsection{6/archdischild-2019-epa.971}

Introduction Arrhythmias in neonates are rare with an incidence reported to be 1 to $5 \%$. Their diagnosis and treatment differs substantially from approaches used in an older child. Through this study we aim to identify epidemiology, clinical features, management and outcome of neonatal arrhythmias.

Patients and methods It's a retrospective study of all cases of arrhythmias hospitalized in the neonatal intensive care unit of Sfax between 2004 and 2018.

Results We registered 10 cases of congenital atrioventricular block (AVB), 8 cases of flutter and 2 cases of chaotic tachycardia. A male predominance was noted with a sex ratio of 2.3. Prenatal diagnosis was performed in 5 cases of atrial flutter by fetal echocardiography which was indicated because of fetal anasarca in 3 cases, a dilatation of the right heart cavities in one case and a fetal tachycardia in one case. Prenatal treatment was administered in 4 cases of atrial flutter. It was based on amiodarone in one case, digoxin in two cases and digoxin associated with sotalol then relayed by flecainide in one case. Ten newborns presented signs of heart failure. For the others, the clinical manifestation was an anomaly of the heart rhythm (bradycardia or tachycardia). Diagnosis was confirmed in all cases by electrocardiogram. Echocardiography was performed for all patients. It showed tight pulmonary narrowing in a case of flutter and transient myocarditis associated to an AVB in one case. After birth, an external electric conversion was necessary in 4 cases of atrial flutter and a pacemaker implantation was indicated in 5 cases of AVB. In the other cases, the treatment was medical (amiodarone for tachycardia and Isoproterenol for AVB). Only 4 newborns with AVB died in the post operative course of the pace maker implantation. For all other newborns the evolution was favorable.

Conclusion Neonatal arrhythmias can be serious and life threatening. The prognosis depends on the prompt of diagnosis which can be challenging at this age. Management must be rapid and multidisciplinary. Hence we insist on the importance of prenatal diagnosis.
P641 MANAGEMENT OF CARDIAC RHABDOMYOMA IN NEONATES

${ }^{1}$ Manel Charfi*, ${ }^{1}$ Rim Zaghdoud, ${ }^{1}$ Amel Ben Hamad, ${ }^{1}$ Chiraz Regaieg, 'Amira Bouraoui, ${ }^{1}$ Ridha Regaieg, ${ }^{1}$ Nedia Hmida, ${ }^{2}$ Dorra Abid, ${ }^{2}$ Samir Kammoun, ${ }^{1}$ Afef Ben Thabet, ${ }^{1}$ Abdellatif Gargouri. 'Department of Neonatology, Hedi Chaker University Hospital, Sfax, Tunisia; ${ }^{2}$ Department of Cardiology Hedi Chaker University Hospital, Sfax, Tunisia

\subsection{6/archdischild-2019-epa.972}

Introduction Cardiac tumors are rare in infant. Most commonly it's a Rhabdomyoma with an incidence of $0.02 \%$ to $0.08 \%$. These cardiac rhabdomyomas are usually multiple with a preferential location in the ventricular myocardium (up to 94\%). We aim to evaluate the epidemiology, clinical features, management and outcome of this rare condition in neonates.

Methods It's a retrospective study of all cases of cardiac rhabdomyomas registered in the neonatal intensive care unit of Sfax between 2009 and 2018.

Results Six full term newborns were included: one boy and five girls. Two of them were twins from a monochorial and monamniotic pregnancy. Prenatal diagnosis was performed in all cases. After birth, all newborns were asymptomatic. Physical exam revealed associated hemangiomas of the forearm in one case and hypomelanotic macules in 4 cases. Postnatal echocardiography confirmed the prenatal findings in all cases. Multiple lesions were noted in 4 cases. Ventricular location was found in all cases. Associated interventricular septum tumor was found in one case as well as a right atrium tumor in one other case. The mean tumor size was $16 * 10 \mathrm{~mm}$. No hemodynamic disorders were noted in all cases. The ophthalmologic and renal assessment was normal for all newborns. Brain MRI was performed in 4 cases. It revealed white matter anomalies and subependymal nodules confirming then the diagnosis of tuberous sclerosis in two patients. During follow up one of them developed focal seizures fully controlled by antiepileptic drugs. The evolution was favorable for all patients. All tumors regressed spontaneously within an average of three years.

Conclusion Cardiac rhabdomyomas are benign tumors which tend to spontaneously regress during early childhood. However they may lead to arrhythmias or hemodynamic disorders due to ventricular inflow/outflow tract obstruction. Thus long term ultrasound follow up is needed. Moreover diagnosis of cardiac rhabdomyoma must lead clinician to look systematically for an associated tuberous sclerosis as it can be its earliest manifestation.

\section{P642 NEONATAL GASTRIC DUPLICATION CYST: A CASE REPORT}

${ }^{1}$ Amel Ben Hamed, 'Manel Charfix, ${ }^{2}$ Hayet Zitouni, ${ }^{1}$ Chiraz Regaieg, ${ }^{1}$ Amira Bouraoui, ${ }^{1}$ Ridha Regaieg, ${ }^{1}$ Nedia Hmida, ${ }^{2}$ Riadh Mhiri, ${ }^{1}$ Afef Ben Thabet, ${ }^{1}$ Abdellatif Gargouri. ${ }^{1}$ Department of Neonatology, Hedi Chaker Hospital, Sfax, Tunisia; ${ }^{2}$ Department of Pediatric Surgery. Hedi Chaker Hospital, Sfax, Tunisia

\subsection{6/archdischild-2019-epa.973}

Background Gastrointestinal duplication(GID) cysts are rare congenital malformations. It occurs approximately in 1 out of 10.000 births. It can occur at any part of the alimentary tract. Gastric duplication cyt constitute 2 to $7 \%$ of all GID. They are usually cystic and located on the greater curvature, 